\title{
The role of prenatal stress as a pathway to personality disorder: Iongitudinal birth cohort study
}

\author{
Ross Brannigan, Antti Tanskanen, Matti O. Huttunen, Mary Cannon, Finbarr P. Leacy and Mary C. Clarke
}

\section{Background}

Many studies have reported associations between prenatal stress and the development of psychotic, anxiety and depressive disorders; however, to date no studies have investigated potential associations with personality disorders.

\section{Aims}

This study investigated potential associations between exposure to prenatal stress and personality disorder in offspring.

\section{Method}

In a subsample $(N=3626)$ of a large Finnish birth cohort, we used logistic regression models to examine associations between selfreported maternal stress during pregnancy, collected monthly during antenatal clinic appointments, and personality disorder in offspring. Familial and outcome information were obtained by linking data from the Finnish Hospital Discharge Register and the Finnish Population Register.

\section{Results}

Compared with those unexposed, children exposed to any maternal stress during gestation had three times the odds of developing a personality disorder (odds ratio $2.76,95 \% \mathrm{Cl}$ $1.59-4.80, P=0.000$ ). Those exposed to moderate stress had three times the odds (odds ratio $3.13,95 \% \mathrm{Cl} 1.42-6.88, P=0.005$ ) and those exposed to severe stress had seven times the odds (odds ratio $7.06,95 \% \mathrm{Cl} 2.10-23.81, \mathrm{P}=0.002$ ) of developing a personality disorder. These associations remained after adjusting for parental psychiatric history, comorbid psychiatric diagnoses, prenatal smoking and antenatal depression.

\section{Conclusions}

Exposure to stress during gestation increases the odds of personality disorder in offspring, independent of other psychiatric disorders. These results suggest the assessment of maternal stress and well-being during pregnancy may be useful in identifying those at greatest risk of developing personality disorder, and highlight the importance of prenatal care for good maternal mental health during pregnancy.

\section{Declaration of interest}

None.

\section{Keywords}

Epidemiology; personality disorders; prenatal stress; prospective study; psychiatry.

\section{Copyright and usage}

(C) The Royal College of Psychiatrists 2019
In recent decades, it has become clear that understanding early life risk factors is vital for the prevention and management of future psychiatric disorders. ${ }^{1}$ Investigations into common psychiatric disorders have highlighted various early life risk factors, such as prenatal stress, social support, maternal anxiety, early life trauma and childhood temperament. ${ }^{1,2}$ However, there is a current gap in the literature regarding potential early life risk factors for personality disorders. Personality disorders have a large personal and societal impact including psychosocial impairment, ${ }^{3}$ increased rates of suicide, $^{4,5}$ functional impairment ${ }^{5}$ and long-term health service use. ${ }^{3,4}$ However, it has also been shown that early intervention is effective in reducing the impact of personality disorder ${ }^{6}$ and, therefore, early identification of those at risk for personality disorder is an important goal. Childhood trauma (including physical and sexual abuse, separation from or loss of parents, and non-secure parentchild bonding) is one of the early life risk factors of personality disorder. $^{7}$ Such factors are usually measured retrospectively which makes them difficult to use as tools for identification of those at risk of developing a personality disorder.

Personality disorder tends to be comorbid with other psychiatric disorders. ${ }^{8}$ Individuals with a prior diagnosis of a personality disorder are significantly more likely to develop other

\footnotetext{
* This article was originally published showing the wrong odds ratio in the abstract and with reversed values in 2 tables. This has now been corrected. The full details of the correction can be found at https://doi. org/10.1192/bjp.2020.50.
}

psychiatric disorders than those without a prior personality disorder diagnosis, and those with a history of mood disorders are more likely to be diagnosed with a personality disorder. Previously, we found a significant association between exposure to prenatal stress and the development of mood disorders. ${ }^{9}$ Several other studies have reported associations between prenatal stress and mental disorders such as depression, ${ }^{10}$ anxiety ${ }^{11}$ and schizophrenia. ${ }^{12}$ Given the lack of specificity in many risk factors (such as prenatal stress exposure) for psychiatric illness, it is plausible that personality disorders share risk factors with other psychiatric disorders. It is important for intervention and prevention strategies in mental illness to know the shared and unique factors that contribute to the risk of developing different outcomes.

In our previous work, ${ }^{9}$ we examined the association between prenatal stress and mental disorders and found that prenatal stress significantly increased the risk of mood disorders. Here we examine the association between prenatal stress and later personality disorder in offspring. This is the first study to examine exposure to prenatal stress as a potential risk factor for the development of personality disorder while controlling for important confounders such as comorbid psychiatric disorders, maternal reported depression during pregnancy, maternal smoking during pregnancy and parental psychiatric history. We hypothesised that exposure to mental stress during gestation would be associated with increased odds of developing a personality disorder, independent of any association with other comorbid psychiatric disorders. 


\section{Method}

\section{Participants}

This study used data from the Helsinki Temperament Cohort, a prospective birth cohort comprising all children born in the greater Helsinki area between 1 July 1975 and 30 June 1976. This cohort $(N=6468)$ was established to examine the effect of prenatal stress and childhood temperament on mental health outcomes. Here we report on a subsample of 3626 cohort members whose mothers completed regular health and well-being assessments during pregnancy.

\section{Ethical approval}

Ethical approval for the collection and use of the Helsinki Temperament Cohort and the corresponding register information was granted by the Ministry of Social Welfare and Health, Finland (approval number 1430/69/82).

\section{Prenatal stress}

Prenatal stress data were collected during monthly antenatal clinic appointments. Women attending the clinic with an expected delivery date between 1 July 1975 and 31 June 1976 were given a booklet by the attending nurse. The booklet contained 15 identical, removable questionnaires on the health and well-being of the mother during the preceding month. The questionnaire directly asked about factors such as subjective stress, subjective feelings of depression, smoking and physical health. Women were asked to complete one questionnaire before each monthly clinic appointment. These prenatal questionnaires included one item on mental stress since the previous visit, and used a three-point Likert scale, with one indicating no stress, two indicating some stress and three indicating notable stress. Individuals could request additional booklets if they attended antenatal clinic appointments more frequently.

\section{Outcome data}

The primary outcome of interest for this study was the diagnosis of personality disorder. Outcome data were obtained through linkage between the Finnish National Population Register and the Finnish Hospital Discharge Register (FHDR). The FHDR was accessed in 2005, at which point the cohort members were approximately 30 years old. All diagnoses recorded in the register were coded using ICD-8 (1967), ICD-9 (1978) or ICD-10 (1992), depending on the date of diagnosis.

\section{Confounders}

We examined the following potential confounders: (a) maternal and paternal psychiatric history from the FDHR, (b) total prenatal questionnaires returned, (c) maternal smoking during pregnancy, (d) maternal report of depression during pregnancy and (e) other psychiatric disorders. Unadjusted and fully adjusted odds ratios are reported.

\section{Parental psychiatric history}

Maternal and paternal psychiatric history could be associated with both the experience of stress during pregnancy and with an increased risk of psychiatric disorder in the offspring. We controlled for both diagnosed maternal and paternal psychiatric disorder to attempt to separate the effects of prenatal stress and parental psychopathology. Paternal psychiatric history information was missing for 73 members of the cohort; these individuals were excluded from the fully adjusted model. Maternal and paternal psychiatric history information was obtained by linking the Finnish National Population Registry and the FHDR.

\section{Number of questionnaires submitted}

There was some variability in the number of prenatal questionnaires returned. Therefore, we adjusted for the numbers of questionnaires returned to account for this.

\section{Prenatal smoking}

Prenatal smoking and prenatal stress have been associated with similar adverse pregnancy outcomes such as low birth weight and preterm delivery as well as psychiatric outcomes such as conduct disorder, attention-deficit hyperactivity disorder and substance misuse. ${ }^{13}$ As individuals who are stressed are also more likely to smoke, ${ }^{14}$ we controlled for smoking during pregnancy in order to ensure prenatal smoking was not driving any observed associations between prenatal stress and personality disorder. Information regarding maternal smoking during pregnancy was also collected as part of the prenatal questionnaires. This information was missing for seven members of the population.

\section{Maternal self-reported feelings of depression}

Many studies examining prenatal stress combine prenatal stress, anxiety and depression into one factor; here we control for maternal feelings of depression during pregnancy in an attempt to isolate the effects of maternal stress from maternal depression during pregnancy. Self-reported maternal depressive symptoms during pregnancy were recorded as part of the monthly prenatal questionnaires. Information on depressive symptoms during pregnancy was missing for 17 women.

\section{Comorbid psychiatric diagnoses}

We adjusted our analysis of the association between prenatal stress and personality disorders for other psychiatric disorders, obtained through the FHDR, to ensure that an association between the exposure and a comorbid disorder did not drive any association seen with personality disorder. In this cohort we previously found significant associations between prenatal stress and mood disorder, as well as an 'any diagnosis' category which included mood disorders, psychotic disorders and anxiety disorders.

\section{Data analysis}

Using Stata $14.2^{15}$ for Windows we ran logistic regression models examining the association between self-reported maternal stress during pregnancy and personality disorder in the offspring. Prenatal stress was recorded using a modal measure of the most frequently reported level of stress throughout pregnancy. A modal measure of stress was used as it best represented the individual scores when compared with the mean, which was less accurate due to variability in the number of returned prenatal questionnaires. If the modal value was not unique, the higher level was used. We used a stepwise method of addition of confounders into our models. This allowed us to assess the impact of each individual confounder as it was added. We also adjusted our main outcome associations for anxiety, mood and psychotic outcomes to ensure any association seen was not driven by associations with these outcomes.

\section{Results}

\section{Descriptive statistics}

In total, 3626 members of the cohort returned at least one prenatal questionnaire; the median number of prenatal questionnaires submitted per woman was six throughout pregnancy, with the lowest 
Table 1. Distribution of exposure to prenatal stress and confounders in those with and without a diagnosis of a personality disorder

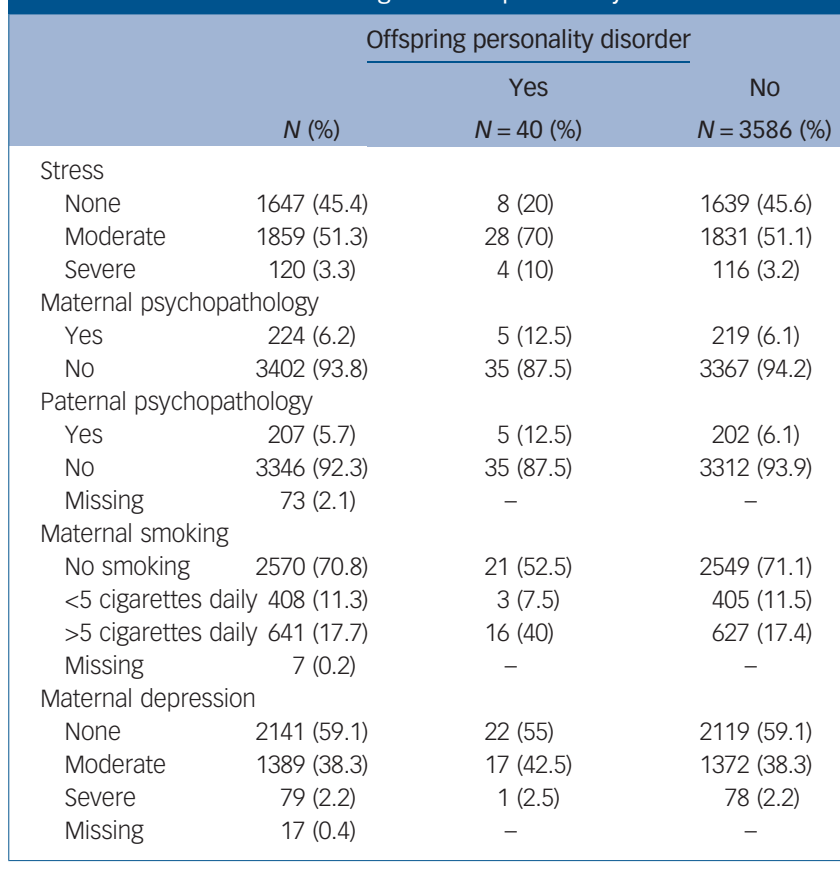

number in early pregnancy. In total there were 40 individuals with personality disorder within the sample of 3626 . There was no significant difference in prevalence of psychiatric outcomes between cohort individuals included in this analysis and those not included. The distribution of reported prenatal stress, personality disorder and the confounders can be seen in Table 1 .

\section{Prenatal stress}

Those who were exposed to either moderate or severe stress during gestation had nearly three times the odds of developing a personality disorder compared with those unexposed to such stress. This increase in odds persisted after controlling for important confounders such as parental psychopathology, maternal smoking during pregnancy, maternal self-reported depression during pregnancy and variation in the number of prenatal questionnaires returned. These associations can be seen in Table 2 .

\section{Comorbidity}

Of the 40 individuals with personality disorder in the sample, 31 had at least one comorbid diagnosis of either a psychotic, depressive or anxiety disorder. In our final analysis we controlled for other individual psychiatric diagnoses, as well as an 'any diagnosis' category in addition to the other confounders. All associations between prenatal stress and personality disorder remain significant after controlling for other comorbid psychiatric disorders (Table 3).

\section{Discussion}

To the best of our knowledge, this is the first study examining the association between maternal stress during pregnancy and the later development of personality disorder in the exposed offspring. Our results suggest that exposure to stress throughout gestation leads to a more than threefold increase in the odds of personality disorder in the offspring after controlling for parental psychopathology, maternal smoking during pregnancy and antenatal depression. When categorising individuals based on the level of stress reported, those exposed to severe stress showed consistently greater odds ratios compared with those exposed to moderate stress, reaching upwards of a ninefold increase in the odds of personality disorder in the offspring. This suggests that the subjective intensity of the experienced stress may moderate the impact of the stress. We also found that the effect of prenatal stress is independent of comorbid psychiatric disorders because the association persisted after adjusting for diagnoses of a depressive, anxiety or psychotic disorder.

These results are in line with previous research examining prenatal stress and its impact on the mental health of offspring. Previously, prenatal stress has been associated with an increased risk of offspring mood disorder, ${ }^{9,10}$ schizophrenia ${ }^{12}$ and anxiety. ${ }^{11}$ These results suggest that prenatal stress is a nonspecific risk factor for mental illness, and also supports current theories suggesting that psychiatric disorders may not be independent constructs but rather share common aetiologies and symptoms, and differ mainly in their expression.

The specific mechanism(s) by which prenatal stress increases the risk of personality disorder are currently unknown. Some potential explanatory factors for the association include biological factors, such as an impact of stress on foetal structural neurodevelopment, and psychosocial factors.

\section{Biological mechanisms}

Studies examining structural brain differences between those with and without personality disorders have shown that those with diagnosed personality disorder have significantly reduced hippocampal and amygdala volumes ${ }^{16}$ as well as significantly reduced volume in the frontal lobe, ${ }^{17}$ left orbitofrontal cortex and right anterior cingulate cortex. ${ }^{16}$ Studies examining the impact of prenatal stress on childhood brain development have found a widespread reduction in grey matter volume throughout brain regions including the prefrontal cortex, premotor cortex, medial temporal lobe, lateral temporal cortex and postcentral gyrus and cerebellum ${ }^{18}$ in middle childhood. Additionally, many patients with personality disorder show hypothalamic-pituitary-adrenal axis dysregulation, including higher resting cortisol levels ${ }^{19}$ and higher cortisol response compared with healthy controls. ${ }^{20}$ This is similar to findings of higher stress cortisol responses in those exposed to prenatal depression and prenatal stress. ${ }^{21,22}$

Genetic predisposition could also play a role in the association between prenatal stress and later mental disorders in offspring. It is possible that the association between prenatal stress and personality disorder in offspring is confounded by shared genetic factors, which may increase the susceptibility to experiencing stress during pregnancy, as well as increasing the odds of personality disorder in offspring. Hannigan and colleagues ${ }^{22}$ found, in a large Norwegian cohort, that shared genetic factors explained approximately $40 \%$ of the associations between prenatal depression and internalising and externalising problems in offspring.

\section{Psychosocial mechanisms}

There may also be many psychosocial factors mediating the relationship between prenatal stress and personality disorder. It is likely that a woman who is stressed during the antenatal period will also be stressed during the postnatal period. It is possible that any continuation of stress into the postnatal period could have an impact on the parent-child relationship, and on early parenting style and warmth, thus affecting the child's development. ${ }^{23}$ This potential pathway has been shown in maternal antenatal depression: In the Avon Longitudinal Study of Parents and Children cohort, Plant $e a^{24}$ found that antenatal depression was significantly associated with maternal postnatal depression, which in turn was associated with maladaptive or nonresponsive parenting. 
Table 2 The association between exposure to prenatal stress and the development of a personality disorder

\begin{tabular}{|c|c|c|c|c|c|}
\hline & $\begin{array}{c}\text { Unadjusted } \\
\text { (odds ratio }[95 \% \mathrm{Cl}])^{\mathrm{a}}\end{array}$ & $\begin{array}{c}\text { Model } 1 \\
(\text { odds ratio }[95 \% \mathrm{Cl}])^{\mathrm{b}}\end{array}$ & $\begin{array}{c}\text { Model } 2 \\
\text { (odds ratio }[95 \% \mathrm{Cl}])^{\mathrm{c}}\end{array}$ & 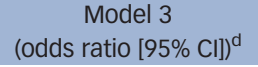 & 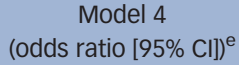 \\
\hline Any stress & $2.76(1.59-4.80)^{*}$ & $2.71(1.55-4.75)^{*}$ & $2.55(1.44-4.52)^{*}$ & $2.34(1.32-4.15)^{*}$ & $3.28(1.75-6.15)^{*}$ \\
\hline Moderate stress & $3.13(1.42-6.89)^{*}$ & $3.10(1.40-6.83)^{*}$ & $2.88(1.30-6.40)^{*}$ & $2.72(1.22-6.06)^{*}$ & $3.91(1.66-9.21)^{*}$ \\
\hline Severe stress & $7.06(2.10-23.81)^{*}$ & $6.76(1.96-23.27)^{\star}$ & $5.99(1.70-21.05)^{*}$ & $4.92(1.37-17.67)^{\star}$ & $9.53(2.36-38.36)^{*}$ \\
\hline \multicolumn{6}{|c|}{$\begin{array}{l}\text { a. Baseline: no stress reported during pregnancy. } \\
\text { b. Adjusted for total prenatal questionnaires returned }(N=3626) \text {. } \\
\text { c. Adjusted for model } 1 \text { and parental psychiatric history }(N=3553) \text {. } \\
\text { d. Adjusted for model } 2 \text { and maternal smoking during pregnancy }(N=3546) \text {. } \\
\text { e. Adjusted for model } 3 \text { and maternal feelings of depression }(N=3532) \text {. } \\
\star P<0.05 \text {. }\end{array}$} \\
\hline
\end{tabular}

\begin{tabular}{|c|c|c|c|c|}
\hline Adjustment made & $\begin{array}{c}\text { Any diagnosis } \\
\text { (odds ratio }[95 \% \mathrm{Cl}] \text { ) }\end{array}$ & $\begin{array}{l}\text { Psychosis diagnosis } \\
\text { (odds ratio }[95 \% \mathrm{Cl}] \text { ) }\end{array}$ & $\begin{array}{c}\text { Depression diagnosis } \\
\text { (odds ratio [95\% Cl]) }\end{array}$ & $\begin{array}{l}\text { Anxiety diagnosis } \\
\text { (odds ratio }[95 \% \mathrm{Cl}] \text { ) }\end{array}$ \\
\hline Any stress & $3.37(1.61-7.07)^{* * * *}$ & $3.32(1.73-6.39)^{\star * * *}$ & $3.11(1.53-6.29)^{* * *}$ & $3.67(1.81-7.41)^{* * * *}$ \\
\hline Moderate stress & $3.54(1.39-8.97)^{\star *}$ & $3.85(1.62-9.18)^{\star * *}$ & $3.65(1.45-9.17)^{\star *}$ & $3.59(1.47-8.78)^{\star *}$ \\
\hline Severe stress & $10.79(2.15-54.13)^{\star * *}$ & $9.79(2.31-41.54)^{\star * *}$ & $8.23(1.69-40.07)^{\star * *}$ & $13.89(3.09-60.56)^{\star * * *}$ \\
\hline
\end{tabular}

Plant and colleagues ${ }^{25}$ also found that individuals exposed to maternal prenatal depression were 2.3 times more likely to have experienced childhood maltreatment and 3.4 times more likely to be diagnosed with depressive disorder. Associations between maternal antenatal depression and childhood maltreatment were replicated in the Avon Longitudinal Study of Parents and Children cohort. ${ }^{24}$ Both studies found that childhood maltreatment independently predicted later psychopathology, further supporting a continuum of early life adverse exposures, where prenatal factors may affect parental-child relationships and thus affect child development. We found that the association between prenatal stress and personality disorder in offspring was independent of maternal prenatal depression. It is possible that measuring women's levels of subjective stress during pregnancy is a good indicator of the psychosocial aspects of the subsequent rearing environment of the child and therefore may be a better measure of the risk to the mental well-being of offspring than the mother's experience of antenatal depression.

Other psychosocial factors, which may mediate the relationship between prenatal stress and personality disorder, include early life separation from parents, childhood trauma and parenting styles. ${ }^{7}$ Various studies have examined the association between child-parent attachment and later borderline personality disorder (BPD). In a review examining Bowlby's ${ }^{26}$ theory of attachment and BPD, Levy ${ }^{27}$ reported that patients with BPD were less likely to report secure attachment to their parent(s) and were more likely to be categorised as having a preoccupied or unresolved attachment to parents.

\section{Strengths and limitations}

We extracted our outcome data from the FHDR which has been found to have excellent diagnostic validity when compared with DSM criteria. ${ }^{28}$ However, because this database only captures the most severe cases of personality disorder (those which require admission to hospital), it may underrepresent the number of individuals with personality disorder in our sample.

This study is unique in using subjectively reported maternal stress collected prospectively on a monthly basis throughout pregnancy. Most previous studies have used the occurrence of specific traumas as the exposure for stress studies. We think that subjective stress is a more accurate quantification of the impact of stress on a woman and her pregnancy.
Additionally, maternal stress, depression and anxiety during pregnancy are often examined in studies as a similar exposure. In this study, we attempted to separate the effects of prenatal stress and depression by adjusting for self-reported depressive symptoms during pregnancy and found an independent significant association between prenatal stress and personality disorder in offspring. We encourage future studies to separate these exposures to shed light on the mechanisms by which stress, depression and anxiety during pregnancy later affect the mental health of the offspring.

We were unable to control for potential confounders such as maternal education, physical and sexual abuse, and familial socioeconomic status. However, we were able to control for important confounders such as parental psychiatric history and antenatal depression. The antenatal depression variable used in this study comprised mother's self-reports of depression symptoms during pregnancy, which were collected as part of the monthly antenatal questionnaires, rather than a depression diagnosis. It is possible that having a different measure of antenatal depression would have led to a stronger association between antenatal depression and offspring psychopathology.

Because of statistical power limitations, we were unable to examine the association between exposure to prenatal stress during different trimesters of pregnancy and personality disorders. It has been previously suggested that there may be a trimester-specific effect of stress exposure on the development of psychiatric disorders, ${ }^{10,11}$ and we suggest that future studies examining the associations between stress and personality disorder also attempt to identify the critical window of exposure during gestation.

Finally, we were unable to split personality disorders into individual disorders or disorder clusters. However, there is debate about the validity of subcategorisation within personality disorder, with evidence suggesting that most people meeting criteria for one personality disorder will meet the criteria for a second. ${ }^{29}$

\section{Future directions of study}

More research is needed to confirm our conclusions and to investigate potential mechanisms involved in the association. The next step in this area of research could involve examinations into whether interventions during pregnancy can reduce the adverse effects of stress. ${ }^{30}$ Some pilot work has shown that intervention during pregnancy can reduce the experience of stress and anxiety. ${ }^{31}$ 
Ross Brannigan (D, BSc, PhD Candidate, Department of Psychology, Royal College of Surgeons in Ireland, Ireland; Antti Tanskanen, PhD, Researcher, Department of Clinical Neuroscience, Karolinska Institute, Sweden; and Department of Mental Health, National Institute for Health and Welfare, Finland; Matti O. Huttunen, PhD, Professor, Department of Mental Health, National Institute for Health and Welfare, Finland Mary Cannon, MD, PhD, Professor, Department of Psychiatry, Royal College of Surgeons in Ireland, Ireland; Finbarr P. Leacy, PhD, Researcher, Data Science Centre, Soy Department of Psychology, Royal College of Surgeons in Ireland; and Department of Psychiatry, Royal College of Surgeons in Ireland, Ireland

Correspondence: Ross Brannigan, Psychology Department, Royal College of Surgeons in Ireland, Room 2, Floor -1, Beaux Lane House, Lower Mercer Street, Dublin 2, Ireland. Email: rossbrannigan@rcsi.com

First received 16 Dec 2018, final revision 26 Jun 2019, accepted 20 Jul 2019

\section{Funding}

Funded by Health Research Board in Ireland, award code HRA-PHR-2015-1130.

\section{References}

1 Rice F, Jones I, Thapar A. The impact of gestational stress and prenatal growth on emotional problems in offspring: a review. Acta Psychiatr Scand 2007; 115: 171-83.

2 Glover V. Annual research review: prenatal stress and the origins of psychopathology: an evolutionary perspective. J Child Psychol Psychiatry 2011; 52: 356-67.

3 Chanen AM, Jovev M, McCutcheon LK, Jackson HJ, McGorry PD. Borderline personality disorder in young people and the prospects for prevention and early intervention. Curr Psychiatry Revi 2008; 4: 48-57.

4 Chanen AM, McCutcheon L. Prevention and early intervention for borderline personality disorder: current status and recent evidence. Br J Psychiatry 2013; 202: s24-9.

5 Pompili M, Girardi P, Ruberto A, Tatarelli R. Suicide in borderline personality disorder: a meta-analysis. Nord J Psychiatry 2005; 59: 319-24.

6 Chanen AM, Jackson HJ, McCutcheon LK, Jovev M, Dudgeon P, Yuen HP, et al. Early intervention for adolescents with borderline personality disorder using cognitive analytic therapy: randomised controlled trial. Br J Psychiatry 2008; 193: 477-84.

7 Bandelow B, Krause J, Wedekind D, Broocks A, Hajak G, Rüther E. Early traumatic life events, parental attitudes, family history, and birth risk factors in patients with borderline personality disorder and healthy controls. Psychiatry Res 2005; 134: 169-79.

8 Plana-Ripoll O, Pedersen CB, Holtz Y, Benros ME, Dalsgaard S, De Jonge P, et al. Exploring comorbidity within mental disorders among a Danish national population. JAMA Psychiatry 2019; 76: 259-70.

9 Brannigan R, Cannon M, Tanskanen A, Huttunen MO, Leacy FP, Clarke MC The association between subjective maternal stress during pregnancy and offspring clinically diagnosed psychiatric disorders. Acta Psychiatr Scand 2019; 139: 304-10.

10 Brown AS, van Os J, Driessens C, Hoek HW, Susser ES. Further evidence of relation between prenatal famine and major affective disorder. Am J Psychiatry 2000; 157: 190-5

11 O'Connor TG, Heron J, Golding J, Glover V, Team ASS. Maternal antenatal anxiety and behavioural/emotional problems in children: a test of a programming hypothesis. J Child Psychol Psychiatry 2003; 44: 1025-36.
12 Khashan AS, Abel KM, McNamee R, Pedersen MG, Webb RT, Baker PN, et al. Higher risk of offspring schizophrenia following antenatal maternal exposure to severe adverse life events. Arch Gen Psychiatry 2008; 65: 146-52.

13 Wakschlag LS, Pickett KE, Cook Jr E, Benowitz NL, Leventhal BL. Maternal smoking during pregnancy and severe antisocial behavior in offspring: a review. Am J Public Health 2002; 92: 966-74.

14 Kouvonen A, Kivimäki M, Virtanen M, Pentti J, Vahtera J. Work stress, smoking status, and smoking intensity: an observational study of 46190 employees. J Epidemiol Community Health 2005; 59: 63-9.

15 StataCorp. Stata Statistical Software: Release 14. StataCorp LP, 2015.

16 Driessen M, Herrmann J, Stahl K, Zwaan M, Meier S, Hill A, et al. Magnetic resonance imaging volumes of the hippocampus and the amygdala in women with borderline personality disorder and early traumatization. Arch Gen Psychiatry 2000; 57: 1115-22.

17 Lyoo IK, Han MH, Cho DY. A brain MRI study in subjects with borderline personality disorder. J Affect Disord 1998; 50: 235-43.

18 Buss C, Davis EP, Muftuler LT, Head K, Sandman CA. High pregnancy anxiety during mid-gestation is associated with decreased gray matter density in 6-9-year-old children. Psychoneuroendocrinology 2010; 35: 141-53.

19 Wingenfeld K, Driessen M, Adam B, Hill A. Overnight urinary cortisol release in women with borderline personality disorder depends on comorbid PTSD and depressive psychopathology. Eur Psychiatry 2007; 22: 309-12.

20 Simeon D, Knutelska M, Smith L, Baker BR, Hollander E. A preliminary study of cortisol and norepinephrine reactivity to psychosocial stress in borderline personality disorder with high and low dissociation. Psychiatry Res 2007; 149: 177-84

21 Entringer S, Kumsta R, Hellhammer DH, Wadhwa PD, Wüst S. Prenatal exposure to maternal psychosocial stress and HPA axis regulation in young adults. Horm Behav 2009; 55: 292-8.

22 Hannigan L, Eilertsen EM, Gjerde LC, Reichborn-Kjennerud T, Eley TC, Rijsdijk FV, et al. Maternal prenatal depressive symptoms and risk for early-life psychopathology in offspring: genetic analyses in the Norwegian Mother and Child Birth Cohort Study. Lancet Psychiatry 2018; 5: 808-15.

23 Bergman K, Sarkar P, Glover V, O'Connor TG. Maternal prenatal cortisol and infant cognitive development: moderation by infant-mother attachment. Biol Psychiatry 2010; 67: 1026-32.

24 Plant DT, Jones FW, Pariante CM, Pawlby S. Association between maternal childhood trauma and offspring childhood psychopathology: mediation analysis from the ALSPAC cohort. Br J PSychiatry 2017; 211: 144-50.

25 Plant DT, Pariante CM, Sharp D, Pawlby S. Maternal depression during pregnancy and offspring depression in adulthood: role of child maltreatment. Br J Psychiatry 2015; 207: 213-20.

26 Bowlby J. The making and breaking of affectional bonds: I. Aetiology and psychopathology in the light of attachment theory. Br J Psychiatry 1977; 130: 201-10

27 Levy KN. The implications of attachment theory and research for understanding borderline personality disorder. Dev Psychopathol 2005; 17: 959-86

28 Isohanni M, Mäkikyrö T, Moring J, Räsanen P, Hakko H, Partanen U, et al. A comparison of clinical and research DSM-III-R diagnoses of schizophrenia in a Finnish national birth cohort. Soc Psychiatry Psychiatr Epidemiol 1997; 32: 303-8.

29 Widiger TA, Rogers JH. Prevalence and comorbidity of personality disorders. Psychiatr Ann 1989; 19: 132-6.

30 Glover V. Maternal depression, anxiety and stress during pregnancy and child outcome; what needs to be done. Best Pract Res Clin Obstet Gynaecol 2014; 28: 25-35

31 Vieten C, Astin J. Effects of a mindfulness-based intervention during pregnancy on prenatal stress and mood: results of a pilot study. Arch Women Ment Health 2008; 11: 67-74 